\title{
Editorial: Developing Bioactive Materials for Dental Applications
}

\author{
Mary Anne Melo ${ }^{1,2 *}$, Fabricio Collares ${ }^{3}$ and Salvatore Sauro ${ }^{4,5}$ \\ ${ }^{1}$ Division of Operative Dentistry, Department of General Dentistry, University of Maryland School of Dentistry, Baltimore, MD, \\ United States, ${ }^{2}$ Dental Biomedical Science PhD Program, University of Maryland School of Dentistry, Baltimore, MD, \\ United States, ${ }^{3}$ Dental Materials Laboratory, Department of Conservative Dentistry, School of Dentistry, Federal University of Rio \\ Grande do Sul, Porto Alegre, Brazil, ${ }^{4}$ Dental Biomaterials and Minimally Invasive Dentistry, Department of Dentistry, Cardenal \\ Herrera-CEU University, CEU Universities, Valencia, Spain, ${ }^{5}$ Department of Therapeutic Dentistry, I. M. Sechenov First Moscow \\ State Medical University, Moscow, Russia
}

Keywords: dental (restorative) composites, biofilm, bioactive, nanotecehnology, biomaterial

\section{Editorial on the Research Topic}

\section{Developing Bioactive Materials for Dental Applications}

Bioactive biomaterials for managing tissue loss due to congenital or acquired diseases as dental caries, periodontitis, and bone defects have gained significant relevance in the last decades. New materials currently being introduced, under development are expected to be bioactive in that they will be intended to interact in some positive way with the oral environment. These materials will provide a wide range of diverse functions, including the inhibition of bacterial biofilm formation, remineralization of mineral loss in dental hard tissues as dentin and enamel, and the regeneration of diseased pulp, bone soft tissues.

The mouth is a dynamic environment, so dental materials regularly experience changes that alter

\section{OPEN ACCESS}

Edited and reviewed by: Hasan Uludag,

University of Alberta, Canada

*Correspondence:

Mary Anne Melo

mmelo@umaryland.edu

Specialty section: This article was submitted to Biomaterials,

a section of the journal

Frontiers in Materials

Received: 01 August 2021 Accepted: 06 August 2021 Published: 18 August 2021

Citation:

Melo MA, Collares $F$ and Sauro $S$ (2021) Editorial: Developing Bioactive

Materials for Dental Applications.

Front. Mater. 8:751618.

doi: 10.3389/fmats.2021.751618 performance, and this highlights the profound need for methods to allow tracking of its performance in physiologically complex.

Generally, dental materials provide core functions, such as mechanical support to masticatory loads (e.g., dental crowns) or optical properties to display a pleasant and natural appearance (e.g., resin composites). This approach has led to the successful design of numerous clinically used materials over the years, such as sealants, orthodontic adhesives, luting cement, hybrids materials for computerassisted design/computer-assisted manufacturing (CAD/CAM)-based restorative dentistry.

Still, advances in material design and polymer chemistry have recently allowed us to incorporate dynamic features into biomaterials. This approach ranges from the design of materials that hold low polymerization shrinkage to eliminate internal stresses and stresses at the restoration's margins to those with stimuli-responsive and interactive properties, where chemical or biological signals can trigger a response in dental material properties or release drugs on-demand. Thus, giving to the material a feature associated with being "biointeractive."

Our increased understanding of native tissue architecture and cell-material interactions and the development of processing methods and chemical syntheses have driven the design of new bioactive agents and, consequently, new biointeractive or bioactive dental materials.

Nanotechnology can provide new solutions in developing dental materials by including bioactive compounds without affecting the functional, aesthetic performance, and other core properties.

Dentistry has an ongoing interest in creating bioactivity for dental materials. Currently, limited dental materials present bioactivity-releasing ions, including those present in tooth minerals. Therefore, the capability of a dental material to positively affect its biological surroundings seems like an avenue for improving the longevity and clinical service inside the mouth. 
The use of bioactive materials, in which either the material itself or a released factor elicits an effect, increases the chances of local regeneration while decreasing the likelihood of adverse effects elsewhere.

Over the years, numerous natural or synthetic nano-to microscale agents have been assessed for bioactivity in the oral environment to be delivered by dental materials in multiple clinical indications. Data to date clearly illustrate that biomaterials for use in the maintenance of oral health are developing at a rapid pace.

In the Research Topic "Developing Bioactive Materials for Dental Applications," inspiring findings on natural and synthetic biomimetic, bioinspired, and bioactive agents and materials developed with its incorporation over different length scales and their capacity to modulate bacterial cell functions and to deliver in localized and sustained fashion various therapeutics were discussed.

As acidogenic bacteria grow over the dental materials used for dental fillings, the tooth-material interface degrades by bacterial acidsleading to increasing premature failure of tooth filling Balhaddad et al. investigated the application of polymerizable antibacterial monomers based on quaternary ammonium compounds as an antibacterial strategy against root caries from high caries risk patients. Feitosa et al. also looked for anti-caries strategies via experimental adhesives containing tailored fluoridated calcium phosphate fillers.

Looking for strategies to enhance the adhesive's interface performance, Spencer et al. develop a molecular-level understanding of a peptide-functionalized adhesive/collagen hybrid biomaterial using Raman spectroscopy combined with a chemometrics approach. An engineered hydroxyapatitebinding peptide (HABP) was copolymerized in dentin adhesive, and dentin was demineralized to provide collagen matrices that were partially infiltrated with the peptidefunctionalized adhesive.

Garcia et al. have shown the promising role of niobium pentoxide filler incorporated into dental adhesives providing good mechanical properties, improved resistance against solvents, and increased radiopacity, without changing the degree of conversion.

Cui et al. explored the polymer-infiltrated ceramic network composites (PICNs) to understand the relationship between surface roughness and flexural strength for CAD-CAM materials. In this work, a novel dental restorative composite was fabricated via infiltrating mixtures of Bis-GMA/TEGDMA and UDMA/TEGDMA into partially sintered porous sodium aluminum silicate blocks and curing.

The pursuit of odontogenic and pulp regeneration approaches has led researchers to investigate different synthesis routes and formulations with combined agents. Jayash et al. revealed the supporting activity in bone healing as a result of the combination of chitosan with different bio-active materials. Chitosan-based gel scaffold and osteoprotegerin-chitosan gel scaffold demonstrated a significant bone quantity and quality compared to unfilled surgical defects in this animal study. Huang et al. highlight the design of mesoporous BG-nanoparticles by a well-established sol-gel method and suggest an odontogenic and acidic-pH neutralizing potential for application in pulp-dentin regeneration under a pathophysiologically challenged acidic environment.

Nassar et al. review the ongoing studies on identifying a cariostatic ability by reducing enamel dissolution of Inositol hexaphosphate (IP6). Inositol is abundant in nature and an essential molecule for different biological functions. In addition, IP6 has a unique structure granting it distinctive properties; a high negative charge density provides IP6 with enormous chelating ability and valuable antioxidant properties. Finally, Jeevanandam et al. presented an outline of recent nanobiomaterials that are extensively investigated for dental implant applications.

As we observe an increasing number of investigations addressing hard dental tissues in the last decade, many efforts are also directed to soft tissues repairs. Canciani et al. analyze the effects of polycaprolactone (PCL) nanofibers enriched with hyaluronic acid and vitamin $\mathrm{E}$ vs nude nanofibers on gingival fibroblasts activity an innovative graft for periodontal soft tissue regeneration purposes. Bermudez et al. looked over the combined use of scaffolds, cells, and bioactive molecules such as peptides are considered the best approach to achieve tissue regeneration. These peptides can induce diverse cellular processes as they can influence cell behavior and modify scaffold properties, giving; as a result, the enhancement of cell adhesion, proliferation, migration, differentiation, and biomineralization that are required given the complex nature of oral tissues.

We hope that this work set will stimulate further research on the development of bioactive materials for dental applications by providing an overview of the latest and most exciting advances in emerging approaches for bioactivity. In addition, this book may help readers to understand the basics and latest developments in this field.

\section{AUTHOR CONTRIBUTIONS}

MM contributed to the writing of the manuscript. FC and SS contributed to the critical review of the manuscript. All authors have read and agreed to the published version of the manuscript.

Conflict of Interest: The authors declare that the research was conducted in the absence of any commercial or financial relationships that could be construed as a potential conflict of interest.

Publisher's Note: All claims expressed in this article are solely those of the authors and do not necessarily represent those of their affiliated organizations, or those of the publisher, the editors and the reviewers. Any product that may be evaluated in this article, or claim that may be made by its manufacturer, is not guaranteed or endorsed by the publisher.

Copyright (c) 2021 Melo, Collares and Sauro. This is an open-access article distributed under the terms of the Creative Commons Attribution License (CC $B Y)$. The use, distribution or reproduction in other forums is permitted, provided the original author(s) and the copyright owner(s) are credited and that the original publication in this journal is cited, in accordance with accepted academic practice. No use, distribution or reproduction is permitted which does not comply with these terms. 